\title{
Pair Distribution Function Analysis in Materials Science
}

\section{Bo Brummerstedt Iversen}

\author{
Aarhus University, Aarhus, Denmark; \\ bo@chem.au.dk
}

X-ray diffraction from powders and single crystals has for decades been the key analytical tool in materials science. Bragg intensities provide information about the average crystals structure, but often it is disorder and specific local structure that control key material properties. For 1D data there has been an immense growth in combined analysis of Bragg and diffuse scattering using the Pair Distribution Function (PDF), and in our group we frequently use 1D PDF analysis to study nanocrystal nucleation in solvothermal processes [1] or thin films [2], or to analyse materials under operating conditions [3]. For single crystals, diffuse scattering studies have a long history with elaborate analysis in reciprocal space, but direct space analysis of the 3D-PDF is still in its infancy. We have used 3D-PDF analysis to study the crystal structures of high performance thermoelectric materials $\mathrm{Cu}_{2} \mathrm{Se}$ (Fig 1) [4], $\mathrm{PbTe}$ [5], and 19e half-heusler $\mathrm{Nb}_{1-\mathrm{x}} \mathrm{CoSb}$ [6], where the true local structure is essential for understanding the unique properties. For frustrated magnetic materials direct space analysis of diffuse magnetic scattering provides a new route to magnetic structures [7].

[1] N. L. N. Broge et al., Auto-catalytic formation of high entropy alloy nanoparticles, Angew. Chem. Intl. Ed., 59, 21920-21924 (2020)

[2] M. Roelsgaard et al., Time-Resolved Surface Pair Distribution Functions during Deposition by RF Magnetron Sputtering, IUCrJ, 6, 299-304 (2019)

[3] L. R. Jørgensen et al., Operando X-ray scattering study of thermoelectric $\beta$ - $\mathrm{Zn}_{4} \mathrm{Sb}_{3}, I U C r-J, 7,100-104$ (2020)

[4] N. Roth et al., Solving the disordered structure of $\beta-\mathrm{Cu}_{2-x}$ Se using the three-dimensional difference pair distribution function, Acta Crystallogr. Sect. A, 75, 465-473 (2019)

[5] K. A. U. Holm et al., Temperature Dependence of Dynamic Dipole Formation in PbTe, Phys. Rev. B, 102, 024112 (2020)

[6] N. Roth et al., A simple model for vacancy order and disorder in defective half-Heusler systems, IUCrJ, 7, 673-680 (2020)

[7] N. Roth et al., Model-free reconstruction of magnetic correlations in frustrated magnets, IUCr-J, 5, 410-416 (2018)

\section{Keywords: 3D-PDF, local structure, nucleation, frustrated magnetism}

